\title{
Reparación primaria con ancla bioabsorbible de ligamento cruzado anterior en avulsión tibial postraumática
}

\author{
Primary repair with bioabsorbible anchor of anterior cruciate \\ ligament in post-traumatic tibial avulsion
}

Muñoz-Galguera RA,* Gómez-Espíndola JC*

Centro Médico Naval.

RESUMEN. Introducción: Las lesiones del ligamento cruzado anterior son comunes; sin embargo, las avulsiones tibiales en adultos son raras. El estándar de oro continúa siendo la reconstrucción, ya sea por aloinjerto o injerto autólogo. En los últimos años se han reportado reparaciones primarias en lesiones de lado femoral mediante anclaje bioabsorbible. Reporte de caso: Presentamos el caso de una reparación primaria de ligamento cruzado anterior por avulsión tibial en un paciente de 19 años, posterior a presentar trauma directo con lesiones agregadas de ligamento cruzado posterior y esquina posterolateral. Se realizó reparación primaria por portal transtendón con sutura del ligamento cruzado anterior en cruz mediante FiberTape y FiberWire con anclaje SwiveLock 4.75 mm en huella tibial. Resultados: A 12 meses de operado se encuentra con marcha independiente, arcos completos, con escalas de valoración clínica, Tegner antes y posterior a la lesión en 6, escala de Lysholm 91, IKDC subjetivo 73.6, IKDC objetivo en C y EQ-5D 0.79. Conclusión: La fijación con ancla bioabsorbible es posible en la avulsión de la espina tibial postraumática.

Palabras clave: Espina tibial, avulsión, ancla, reparación, primaria, ligamento.

\section{Introducción}

La evolución del tratamiento del ligamento cruzado anterior (LCA) inició en 1895, cuando Mayo Robson reportó una reparación primaria en una lesión de LCA en un pa-
ABSTRACT. Introduction: Anterior cruciate ligament injuries are common, however, tibial avulsions in adults are rare. The gold standard continues to be reconstruction either by allograft or autologous graft. Primary repairs in femoral side lesions have been reported in recent years by bioabsorbable anchors. Case report: We present the case of a primary repair of anterior cruciate ligament due to tibial avulsion in a patient 19 years after presenting direct trauma with added lesions of posterior cruciate ligament and posterolateral corner. Primary repair was performed by trans-tendon portal with suture of the anterior cruciate ligament crossed by FiberTape and FiberWire with a SwiveLock anchor $(4.75 \mathrm{~mm})$ in tibial footprint. Results: Twelve months after surgery is with independent gait, full range of movement, with clinical rating scales, Tegner before injury and post-injury at 6, Lysholm 91, 73.6 subjective IKDC, objective IKDC in C and EQ-5D 0.79. Conclusion: Fixation with bioabsorbable anchor is possible in the avulsion of the post-traumatic tibial spine.

Keywords: Tibial spine, avulsion, anchor, repair, primary, ligament.

* Servicio de Traumatología y Ortopedia, Centro Médico Naval, Secretaría de Marina-Armada de México. Ciudad de México.

Dirección para correspondencia:

Ruperto Alfonso Muñoz-Galguera

Avenida Heroica Escuela Naval Militar Núm. 745, Col. Presidentes Ejidales, CP 04470,

Alcaldía de Coyoacán, Ciudad de México, México.

E-mail: ruperto.alfonso.m.g@gmail.com

Citar como: Muñoz-Galguera RA, Gómez-Espíndola JC. Reparación primaria con ancla bioabsorbible de ligamento cruzado anterior en avulsión tibial postraumática. Acta Ortop Mex. 2020; 34(4): 238-241. https://dx.doi.org/10.35366/97558

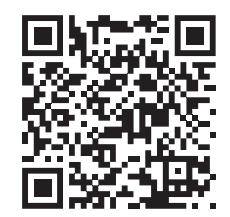


LCA en la década de los 70 y 80 con buenos resultados a corto plazo. ${ }^{3,4,5,6}$ Sin embargo, a mediano plazo los resultados se deterioraban, como demostraron Feagin y Curl en 1976 con 53\% de reincidencia en rupturas a los cinco años de seguimiento con altos índices de dolor, rigidez e inestabilidad. ${ }^{6}$

Se sumaron varios ensayos clínicos prospectivos aleatorizados demostrando mejores resultados en seguimiento de reconstrucción de LCA, en comparación con las reparaciones primarias. Esto resultó en el abandono de la reparación primaria abierta como tratamiento de la lesión de LCA al principio de la década 90 y dejando como el nuevo estándar la reconstrucción del LCA para todos los pacientes. $7,8,9,10,11,12$

Sin embargo, cuando el ligamento nativo es preservado se asume que la cirugía es menos invasiva, tiene algunas ventajas sobre la reconstrucción del LCA, incluyendo mantener la propiocepción y prevención de las complicaciones de la toma del injerto. ${ }^{13,14,15,16,17}$ En 1991, Sher$\operatorname{man}^{18}$ reportó los diferentes tipos de ruptura del LCA, tipo I avulsión femoral, tipo 2 con $20 \%$ de tejido femoral, tipo 3 con $30 \%$ de tejido femoral y tipo 4 con lesión a nivel de $50 \%$ del ligamento, el cual se estima que sucede en $70-90 \%$.

La técnica quirúrgica de las reparaciones primarias en la década de los 80 y 90 se basa en artrotomías, lo que se considera actualmente como un método más invasivo y con mayor morbilidad cuando se comparan con las técnicas artroscópicas. Se intuye que esto contribuyó a los resultados subóptimos en esas décadas, agregando la inmovilización prolongada. $^{2}$

El primer estudio artroscópico en una reparación primaria de LCA fue realizado por DiFelice y colaboradores, ${ }^{19}$ quienes reportaron una serie de casos de 11 pacientes con

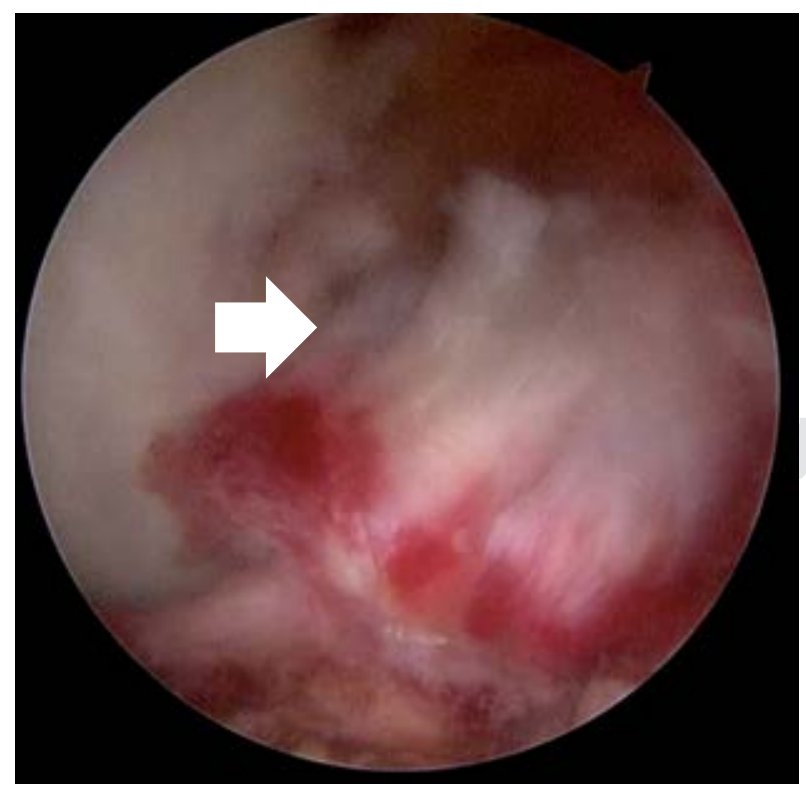

Figura 1: Se observa ruptura del LCA en su lado tibial.

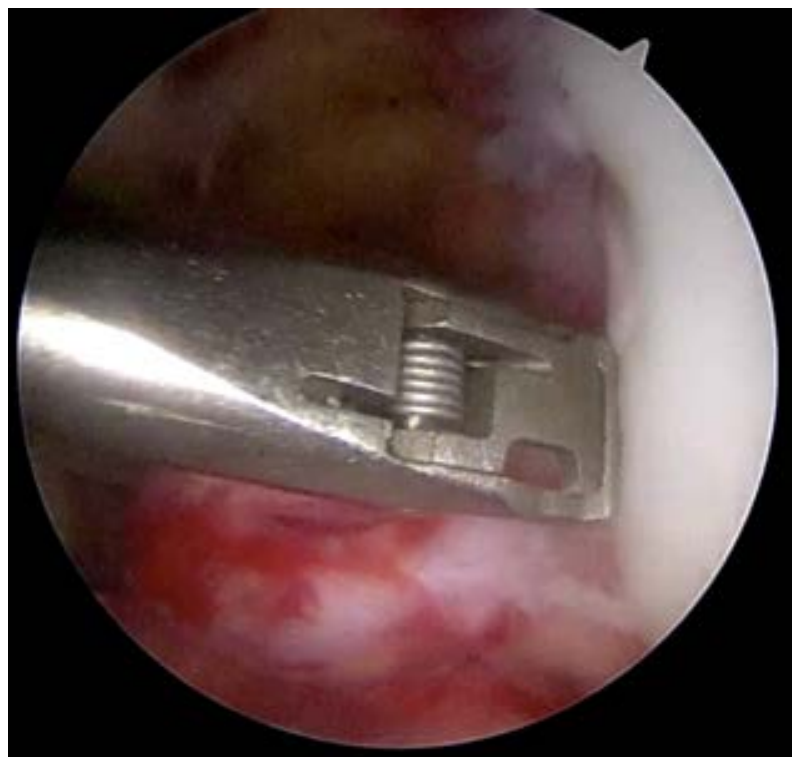

Figura 2: Por medio de la pinza y aguja Scorpion se sujeta ligamento en plano transversal.

rupturas proximales y tratados con sutura con anclaje artroscópico. Reportando sólo una falla (9\%) con un KT-1000 de $6 \mathrm{~mm}$ de diferencia.

\section{Caso clínico}

Masculino de 19 años de edad que es traído al Servicio de Urgencias al ser atropellado por vehículo automotor, refiriendo dolor de gran intensidad en extremidad inferior izquierda. A la exploración física se observa rodilla izquierda con aumento de volumen y presencia de dermoabrasión en cara lateral, limitación importante a la movilización activa y pasiva, el control radiológico en dos proyecciones con congruencia articular sin evidencia de fracturas. Se colocó vendaje algodonoso y férula posterior para evaluación dos semanas después con resonancia magnética. En la nueva valoración se encontró arco de movilidad: flexión a $90^{\circ}$ dolorosa, extensión completa, con cajón anterior y posterior positivo, signo de Lachman positivo y estrés en valgo positivo. Resonancia magnética de rodilla izquierda reportó lesión de ligamento cruzado anterior, posterior y ligamento colateral lateral. Se programó para artroscopía de rodilla izquierda y reconstrucción ligamentaria. Al recorrido artroscópico se observó ruptura total de ligamento cruzado posterior (LCP), avulsión de LCA en inserción tibial (Figura 1), sinovitis y hematoma residual; se realizó portal anteromedial y portal posteromedial bajo visión directa, se realizó limpieza de remanente de LCP y posteriormente reconstrucción con aloinjerto de $9 \mathrm{~mm}$ de diámetro con fijación femoral con TightRope (Arthrex) y a nivel tibial con tornillo biocompuesto $10 \times 30 \mathrm{~mm}$ (Arthrex). Posteriormente se realizó la reparación de LCA por portal transtendón en inserción tibial mediante paso de sutura en cruz a través del ligamento con FiberWire y Tiger- 
Tape (Arthrex) con aguja Scorpion (Arthrex) (Figuras 2 y 3) y posterior a limpieza de huella tibial se fija con ancla SwiveLock, observando reparación satisfactoria (Figura 4). Después se realizó abordaje en palo de jockey en cara lateral de rodilla de $10 \mathrm{~cm}$, se disecó por planos hasta fascia lata, se identifica bíceps y ventana posterolateral. Se observa ruptura completa de ligamento colateral lateral y se procede a tunelizar de anterior a posterior cabeza de peroné, se pasa aloinjerto de $7 \mathrm{~mm}$ y se fijan las dos bandas en sitio de inserción femoral del ligamento colateral lateral con tornillo biocompuesto de $8 \times 23 \mathrm{~mm}$.

Posterior al procedimiento quirúrgico se colocó rodillera mecánica bloqueada en extensión durante tres semanas, después se inició flexión a 30 grados y se fue aumentando cada semana 30 grados hasta lograr 120 grados a la octava semana. Se mantuvo sin apoyo de extremidad por cuatro semanas, inició apoyo parcial progresivo y carga completa a las seis semanas. Las primeras cuatro semanas se trató por especialista en rehabilitación con ultrasonido y electroterapia tres sesiones por semana. El apoyo parcial y la reeducación del patrón de marcha se desarrolló en barras paralelas, a la sexta semana dio inicio uso de tanque terapéutico con inmersión por niveles para mejorar fuerza muscular y arco de movilidad de toda la extremidad. El retiro de rodillera se hizo a la octava semana.

A 12 meses de evolución, el paciente tenía marcha independiente. Arco de movilidad con extensión a 0 y flexión a 120 grados; ligero cajón anterior comparativo al lado sano, sin cajón posterior y con adecuada estabilidad en varo y valgo. A la medición con rolímetro se encontró una diferencia de $4 \mathrm{~mm}$ a la rodilla sana. Las escalas de valoración clínica con el mismo nivel de escala de actividad de Tegner antes de la lesión y posterior a la misma en nivel 6. La escala de Lysholm con 91 puntos, IKDC subjetivo de 73.6, IKDC ob-

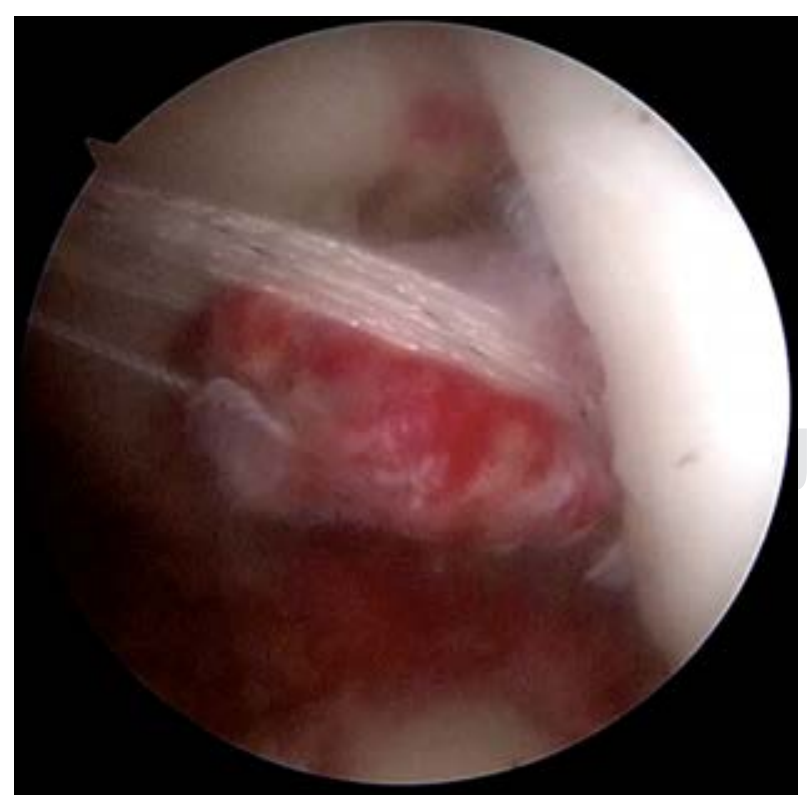

Figura 3: Imagen del LCA con las dos suturas colocadas.

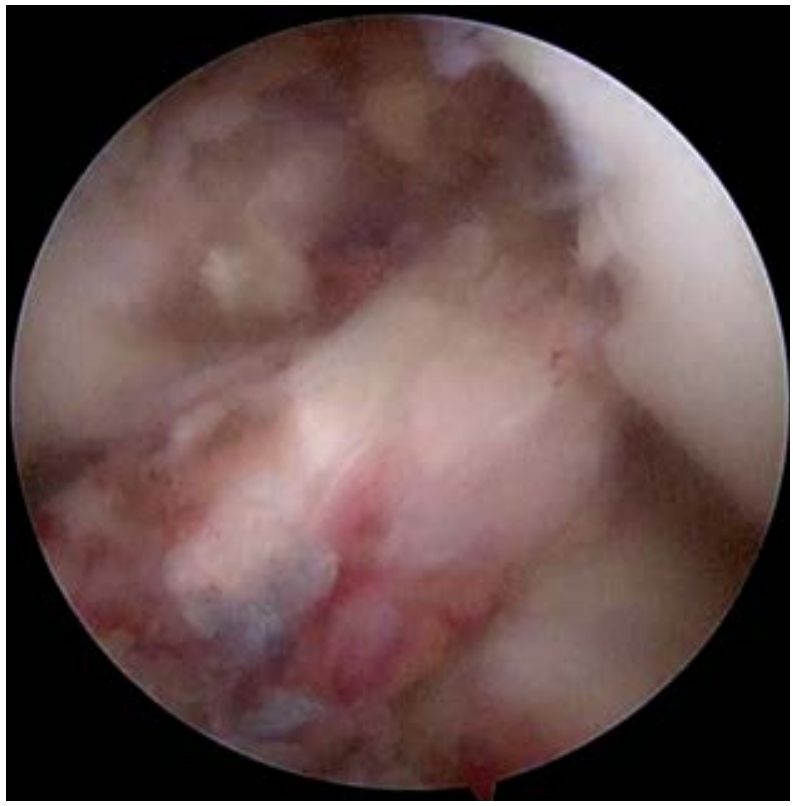

Figura 4: LCA reinsertado.

jetivo en $\mathrm{C}$ y, por último, en el EQ-5D nos dio un resultado en calidad de vida de 0.79 . Resonancia magnética de control a 12 meses con integridad de ligamento cruzado anterior y adecuada dirección de sus fibras (Figura 5).

\section{Discusión}

Las avulsiones distales del ligamento cruzado anterior en adultos son raras. Si bien la reparación primaria está resurgiendo, las reparaciones por avulsión tibial no se han reportado de forma frecuente. Ahn en 2012 reportó su técnica con tres túneles transóseos tibiales y sutura al LCA en un masculino de 17 años con muy buenos resultados posteriores a la reparación. ${ }^{20}$ Sheth y colaboradores, en 2016, reportan por misma técnica la reparación primaria por medio de sutura del ligamento y fijación a la huella tibial a través de dos túneles transóseos con fijación a la cortical tibial por medio de un tornillo metafisario o anudando las suturas, reportando adecuada evolución radiológica a los seis meses. ${ }^{21}$

En nuestro caso, optamos por el concepto de DiFelice ${ }^{19}$ en rupturas de LCA en su lado femoral con fijación mediante ancla bioabsorbible y posterior a 12 meses hay adecuada evolución tanto imagenológica como clínica. Aunque tenemos una traslación aumentada en el comparativo del rolímetro, el paciente nos refirió satisfacción de acuerdo con sus actividades, tomando en cuenta que es una lesión multiligamentaria.

\section{Conclusión}

La fijación con ancla bioabsorbible es posible en la avulsión de la espina tibial postraumática, en el corto plazo la recuperación es adecuada. 

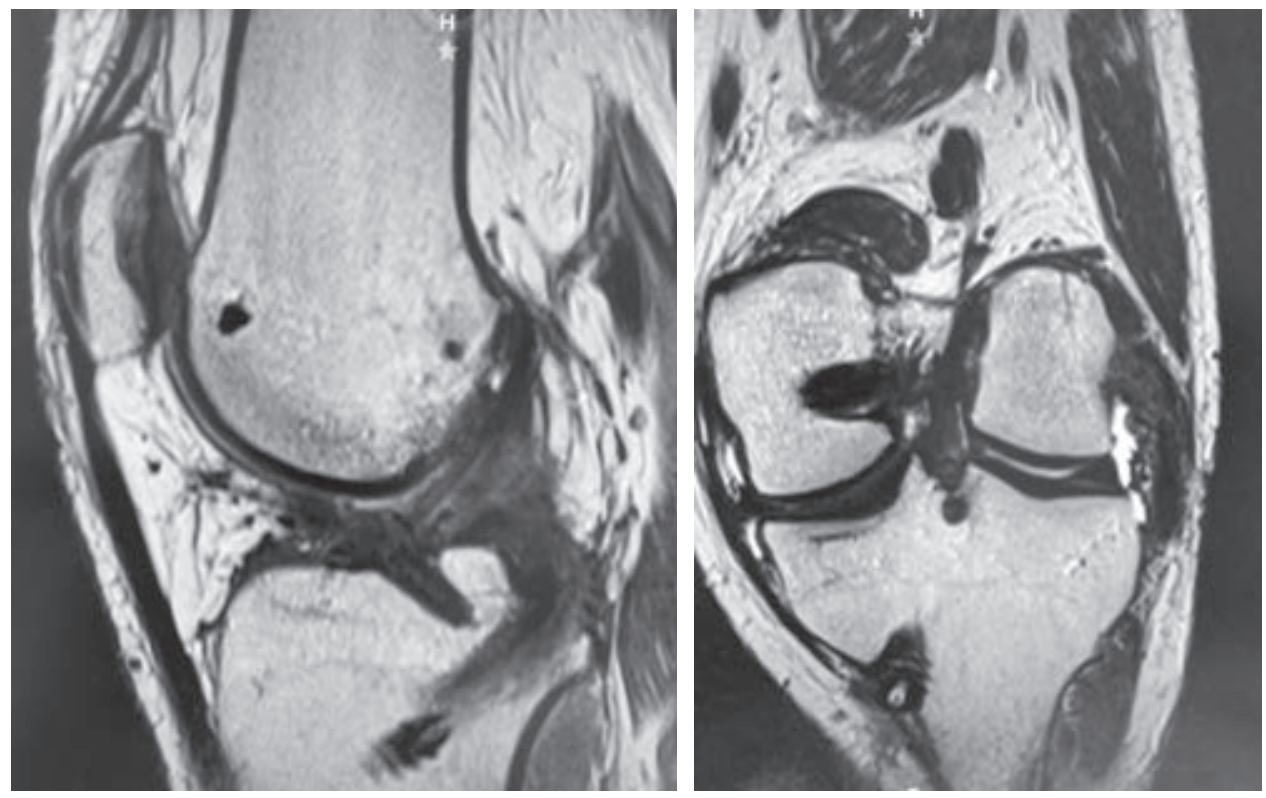

Figura 5:

Resonancia magnética 12 meses postquirúrgica, corte sagital y coronal. Se muestra sitio de colocación de ancla y LCA presente.
Bibliografía

1. Robson AW. VI. Ruptured crucial ligaments and their repair by operation. Ann Surg. 1903; 37(5): 716-8.

2. van der List JP, DiFelice GS. Primary repair of the anterior cruciate ligament: a paradigm shift. Surgeon. 2017; 15(3): 161-8.

3. Palmer I. On the injuries to the ligaments of the knee joint. Acta Chir Scand. 1938; 53.

4. O’Donoghue DH. An analysis of end results of surgical treatment of major injuries to the ligaments of the knee. J Bone Joint Surg Am. 1955; 37-A(1): 1-13; passim.

5. O'Donoghue DH. Surgical treatment of fresh injuries to the major ligaments of the knee. J Bone Joint Surg Am. 1950; 32 A(4): 721-38.

6. Feagin JA Jr, Curl WW. Isolated tear of the anterior cruciate ligament: 5-year follow-up study. Am J Sports Med. 1976; 4(3): 95-100.

7. Andersson C, Odensten M, Good L, Gillquist J. Surgical or nonsurgical treatment of acute rupture of the anterior cruciate ligament. A randomized study with long-term follow-up. J Bone Joint Surg Am. 1989; 71(7): 965-74.

8. Engebretsen L, Benum P, Fasting O, Mølster A, Strand T. A prospective, randomized study of three surgical techniques for treatment of acute ruptures of the anterior cruciate ligament. Am J Sports Med. 1990; 18(6): 585-90.

9. Grøntvedt T, Engebretsen L, Benum P, Fasting O, Mølster A, Strand T. A prospective, randomized study of three operations for acute rupture of the anterior cruciate ligament. Five-year follow-up of one hundred and thirty-one patients. J Bone Joint Surg Am. 1996; 78(2): 159-68.

10. Grøntvedt T, Engebretsen L. Comparison between two techniques for surgical repair of the acutely torn anterior cruciate ligament. A prospective, randomized follow-up study of 48 patients. Scand J Med Sci Sports. 1995; 5(6): 358-63.

11. Odensten M, Hamberg P, Nordin M, Lysholm J, Gillquist J. Surgical or conservative treatment of the acutely torn anterior cruciate ligament.
A randomized study with short-term follow-up observations. Clin Orthop Relat Res. 1985; (198): 87-93.

12. Sandberg R, Balkfors B, Nilsson B, Westlin N. Operative versus nonoperative treatment of recent injuries to the ligaments of the knee. A prospective randomized study. J Bone Joint Surg Am. 1987; 69(8): 1120-6.

13. Dhillon MS, Bali K, Prabhakar S. Differences among mechanoreceptors in healthy and injured anterior cruciate ligaments and their clinical importance. Muscles Ligaments Tendons J. 2012; 2(1): 38-43.

14. Barrett DS. Proprioception and function after anterior cruciate reconstruction. J Bone Joint Surg Br. 1991; 73(5): 833-7.

15. Busam ML, Provencher MT, Bach BR Jr. Complications of anterior cruciate ligament reconstruction with bone-patellar tendon-bone constructs: care and prevention. Am J Sports Med. 2008; 36(2): 37994.

16. Aga C, Wilson KJ, Johansen S, Dornan G, La Prade RF, Engebretsen L. Tunnel widening in single- versus double-bundle anterior cruciate ligament reconstructed knees. Knee Surg Sports Traumatol Arthrosc. 2017; 25(4): 1316-27.

17. Griffith TB, Allen BJ, Levy BA, Stuart MJ, Dahm DL. Outcomes of repeat revision anterior cruciate ligament reconstruction. Am J Sports Med. 2013; 41(6): 1296-301.

18. Sherman MF, Lieber L, Bonamo JR, Podesta L, Reiter I. The longterm followup of primary anterior cruciate ligament repair. Defining a rationale for augmentation. Am J Sports Med. 1991; 19(3): 243-55.

19. DiFelice GS, Villegas C, Taylor S. Anterior cruciate ligament preservation: early results of a novel arthroscopic technique for suture anchor primary anterior cruciate ligament repair. Arthroscopy. 2015; 31(11): 2162-71.

20. Ahn JH, Han KY, Yu IS, Koh KH. Arthroscopic treatment for tibial "Peel off" tears in anterior cruciate ligament-case report. Eur J Orthop Surg Traumatol. 2013; 23 Suppl 2: S251-5.

21. Sheth MR, Tapasvi SR, Patil SS. Primary repair of tibial-sided avulsion of the anterior cruciate ligament. Arthrosc Tech. 2016; 5(4): e901-6. 